Annuaire suisse de politique de développement

Partenariats public-privé et coopération internationale

\title{
La cogestion étatique-communautaire de l'eau à Cochabamba (Bolivie)
}

Sabine Hoffmann

\section{OpenEdition}

1 Journals

Édition électronique

URL : http://journals.openedition.org/aspd/362

DOI : 10.4000/aspd.362

ISSN : 1663-9669

Éditeur

Institut de hautes études internationales et du développement

\section{Édition imprimée}

Date de publication : 1 octobre 2005

Pagination : 179-190

ISSN : 1660-5934

\section{Référence électronique}

Sabine Hoffmann, «La cogestion étatique-communautaire de l'eau à Cochabamba (Bolivie) ", Annuaire suisse de politique de développement [En ligne], 24-2 | 2005, mis en ligne le 19 février 2010, consulté le 08 septembre 2020. URL : http://journals.openedition.org/aspd/362 ; DOI : https://doi.org/10.4000/ aspd.362 


\section{La cogestion étatique-communautaire de l'eau à Cochabamba (Bolivie)}

\section{Sabine Hoffmann*}

omme bien d'autres notions couramment utilisées dans le domaine du développement, la notion de «partenariat public-privé (PPP)» recouvre de multiples significations. Afin de tenir compte de l'éventail de significations (générales et particulières), il apparaît indispensable d'éclaircir les différents éléments constitutifs de cette notion, tout en précisant que ceux-ci s'inscrivent dans le temps et l'espace, et donc dans un contexte écologique et social déterminé ${ }^{\text {. }}$.

Dans ce sens, étant donné que les partenariats soulèvent inéluctablement des questions de conditions institutionnelles définissant les droits et les obligations, les privilèges et les non-droits des partenaires, l'article vise à aborder un des éléments constitutifs de cette notion: celui de régimes institutionnels ${ }^{2}$ de propriété et de possession ${ }^{3}$.

Ainsi, l'article cherche à approfondir l'hypothèse que la délégation des droits et des obligations à des organisations privées ne représente qu'une forme de partenariats parmi plusieurs autres, à commencer par le partenariat entre organisations étatiques et communautaires. En outre, il vise à clarifier que ces différentes organisations peuvent être soumises aussi bien à la rationalité économique (liée aux régimes de propriété) qu'à la raison écosociale (liée aux régimes de possession). Afin d'illustrer cette diversité de régimes et de logiques, l'article présente l'étude de cas d'un partenariat à Cochabamba, en Bolivie, visant à approvisionner la zone sud de cette ville en eau potable.

\section{Les conditions institutionnelles des partenariats public-privé}

$\mathrm{Au}$ sens strict, les institutions sont des règles collectives qui définissent la marge de comportement des individus au sein d'une société donnée ${ }^{4}$. En définissant ce

* Doctorante à l'IUED, National Centre of Competence in Research (NCCR) North-South. Cet article s'inspire de la conférence «Propriété ou possession: vers une alternative à Cochabamba ?» donnée le 19 mars 2005 par Pascal van Griethuysen et Sabine Hoffmann dans le cadre du Forum alternatif mondial de l'eau, à Genève.

1 Rolf Steppacher, «La résistible imprécision des notions économiques», L'économie à la recherche du développement. Crise d'une théorie, violence d'une pratique, Nouveaux Cahiers de l'IUED, $n^{\circ} 5$, Genève, IUED; Paris, Presses Universitaires de France, 1996, pp. 39-52.

2 Daniel Bromley, Environment and Economy: Property Rights and Public Policy, Oxford, Blackwell, 1991.

3 Gunnar Heinsohn, Otto Steiger, Eigentum, Zins und Geld. Ungelöste Rätsel der Wirtschaftswissenschaften, Marburg, Metropolis, 2002 [1996].

4 Daniel Bromley, Economic Interests and Institutions: The Conceptual Foundations of Public Policy, Oxford, Blackwell, 1989, p. 44. 
qui est socialement acceptable et ce qui ne l'est pas, elles déterminent également ce qui constitue des droits et des obligations, des privilèges et des non-droits, établissant ainsi les relations entre les individus. L'accent est alors mis sur la corrélation entre droits et obligations: les droits des individus ne sont assurés que dans la mesure où ils sont garantis par l'obligation des autres de les respecter, et donc dans la mesure où il existe une autorité dotée de la volonté et de la capacité de l'assurer.

Cette définition des institutions met en évidence que les organisations obtiennent leur signification des institutions: les organisations n'existent que dans la mesure où il y a un ensemble de règles qui définit ce qui est une organisation et ce qui ne l'est pas ${ }^{5}$. Ainsi, les organisations telles que définies par les institutions représentent des sous-groupes sociaux dont l'ensemble des individus qui les composent se donne une structure interne qui est distincte de la structure sociale de la société qui l'intègre ${ }^{6}$.

Finalement, les régimes institutionnels de propriété peuvent être définis comme des structures de droits et d'obligations, de privilèges et de non-droits, qui déterminent les relations entre les individus par rapport à une ressource (ou un bien) ${ }^{7}$. Bromley distingue quatre principaux types de régimes institutionnels de propriété: 1) les régimes de propriété étatique; 2) les régimes de propriété privée; 3) les régimes de propriété communautaire; 4) l'accès ouvert. Afin de différencier les divers droits dont les individus jouissent au sein de chaque régime, Schlager et Ostrom $^{8}$ proposent de distinguer cinq principaux droits de propriété: droits d'accès, d'usage, de gestion, d'exclusion et d'aliénation de ressources ou de biens.

En principe, au sein d'un régime de propriété étatique, le détenteur des droits est l'Etat; celui-ci peut aussi bien exercer les différents droits directement (à travers des organisations étatiques) que les transférer pendant un temps limité aux autres individus ou groupes (organisations privées ou communautaires).

A l'opposé, au sein d'un régime de propriété privée, les droits de propriété restent dans les mains des individus (propriété individuelle) ou des groupes (propriété corporative), l'exclusion des non-détenteurs de droits constituant le trait distinctif de ce type de régime.

En principe, le régime de propriété communautaire, en excluant également tous les non-détenteurs de droits, représente pour le groupe de détenteurs de droits un régime de propriété privée. Cependant, au sein du régime de propriété communautaire, les individus détiennent des droits et des obligations spécifiques. Ces groupes varient en termes de nature, de dimension et de structure interne. Pourtant, ils représentent des unités sociales avec des membres et des frontières bien définis, certains intérêts en commun, quelques interactions entre les membres, et certaines valeurs et normes partagées ${ }^{9}$. En outre, les comportements des membres du groupe sont soumis à des règles acceptées par et visibles

5 Ibid., p. 43.

6 Rolf Steppacher, «Ecologie globale et développement», notes de cours, 14/12/2000, 18 et 25/01/2001, Genève, IUED, semestre d'hiver 2003/2004.

7 Daniel Bromley, Environment and Economy: Property Rights and Public Policy, Oxford, Blackwell, 1991.

8 Edella Schlager, Elinor Ostrom, «Property-Rights Regimes and Natural Resources: A Conceptual Analysis », Land Economics, vol. 68, n 3, 1992, pp. 249-262.

9 Daniel Bromley, Environment and Economy..., op. cit., pp. 26-27. 
pour l'ensemble des individus. L'observation de ces règles est souvent assurée par des systèmes d'autorité endogènes, ce qui constitue l'un des traits distinctifs de ce type de régime ${ }^{10}$.

Selon Bromley, la différence entre régimes de propriété privée et régimes de propriété communautaire ne se trouve pas dans la nature des droits et des obligations, mais plutôt dans leur distribution, et plus particulièrement dans la manière dont le groupe gère l'inclusion et l'exclusion des individus. Cette différence se manifeste entre autres dans la réticence du groupe à expulser des individus lorsque cette expulsion entraînera la détresse de ces derniers. Dans ce sens, le groupe s'accorde sur le fait d'abaisser son propre niveau de vie en commun plutôt que d'exclure des individus.

Néanmoins, le régime de propriété communautaire n'est pas à confondre avec le régime d'accès ouvert. Le trait distinctif de ce dernier est l'absence de propriété (res nullius), et donc l'absence des droits et des obligations des individus par rapport à une ressource ou à un bien particulier. Cette absence remonte à l'absence, à la carence ou à l'effondrement d'un système d'autorité.

Or, dans le cadre d'une théorie économique fondée sur la propriété, Heinsohn et Steiger ${ }^{11}$ proposent de distinguer les régimes de propriété des régimes de possession. Bien que ces deux régimes institutionnels puissent être attribués aussi bien à des organisations étatiques et privées qu'à des organisations communautaires, c'est le type d'évaluation économique à laquelle ils conduisent qui les distingue fondamentalement.

En principe, le régime de possession établit des droits d'usage physique envers des ressources ou des biens (droits de possession). Autrement dit, il définit qui, dans une société donnée, a le droit d'utiliser quel type de ressources ou de biens, comment, quand, pour combien de temps, où, sous quelle réserve et à l'exclusion de qui. Selon Steppacher ${ }^{12}$, ce régime repose sur une raison écosociale qui vise à balancer l'ensemble de ressources ou de biens disponibles dans un contexte écologique donné afin de satisfaire tous les besoins minimaux des êtres humains concernés dans un contexte social déterminé, en faisant recours aux valeurs et aux savoirs culturellement spécifiques.

Par contre, le régime de propriété établit des droits de possession et ajoute à ceux-ci des titres de propriété. Ces titres entraînent de nouveaux droits d'usage non physique des ressources ou des biens (droits de propriété), à commencer par le droit d'engager les titres de propriété comme sécurité dans une relation de crédit, contrat constitutif d'une économie monétaire de propriété ${ }^{13}$.

L'exercice de ce droit d'entrer dans des relations de crédit par des organisations (étatiques, privées et communautaires) conduit inéluctablement à l'endettement de ces dernières. C'est précisément cet endettement qui entraîne la rationalité

10 Ibid.

11 Gunnar Heinsohn, Otto Steiger, Eigentum, Zins und Geld. Ungelöste Rätsel der Wirtschaftswissenschaften, Marburg, Metropolis, 2002 [1996].

12 Rolf Steppacher, groupe de travail «Propriété et possession», Genève, IUED, 05/05/2005. Voir aussi Rolf Steppacher, «La résistible imprécision des notions économiques», in Christian Comeliau (dir.), L'économie à la recherche du développement. Crise d'une théorie, violence d'une pratique, Nouveaux Cahiers de l'IUED, nº 5, Genève, IUED; Paris, Presses Universitaires de France, 1996, pp. 39-52.

13 Gunnar Heinsohn, Otto Steiger, op. cit. 
économique particulière de l'économie de propriété: obligées de s'acquitter de l'obligation contractuelle de rembourser le crédit et de payer un intérêt sous forme monétaire, les organisations engagées dans des relations de crédit sont forcées de demeurer solvables. Cela concerne également les organisations étatiques et communautaires lorsque celles-ci, incapables de financer leurs activités économiques par des revenus et des impôts (organisations étatiques) ou par des contributions monétaires et non monétaires de leurs membres (organisations communautaires), recourent à des crédits. Dès lors, ces organisations sont obligées de soumettre l'ensemble des transactions liées à la réalisation des activités économiques (financées par le crédit) à une évaluation monétaire.

Contraintes de rembourser la dette avec un intérêt, elles sont obligées d'assurer que ces activités économiques soient rentables, raison pour laquelle ces activités sont soumises à une analyse coûts-bénéfices en termes monétaires ${ }^{14}$ qui vise à réduire les coûts et à maximiser les revenus monétaires. Par conséquent, tous les besoins qui ne s'expriment pas dans le marché sous forme d'un pouvoir d'achat, manifestant ainsi une demande solvable, sont désormais négligés ${ }^{15}$. Dès lors, les considérations sociales sont soumises aux exigences de la solvabilité et de la rentabilité, qui entraînent la rationalité économique particulière des régimes de propriété ${ }^{16}$.

Pour conclure, il convient de souligner que chaque régime de propriété ou de possession repose sur des droits spécifiques qui demandent d'être clarifiés dans un contexte écologique et social donné. En outre, il est important d'indiquer que, bien que les différentes organisations (étatiques, privées ou communautaires) soient soumises aux régimes de propriété ou de possession, cela n'exclut pas qu'elles puissent être guidées par une raison sociale ou par une rationalité économique. Evidemment, les associations ici faites ne représentent que des tendances qui nécessitent d'être précisées à la lumière d'un contexte déterminé.

\section{La cogestion étatique-communautaire à Cochabamba: exemple d'un partenariat}

\section{Le contexte écologique et social}

La ville de Cochabamba a subi une croissance démographique accélérée au cours des dernières décennies. En effet, sa population est passée de 75'000 habitants en 1950 à $220^{\prime} 000$ en 1975 , pour arriver à plus de $575^{\prime} 000$ habitants en 2004. La majeure partie de cette croissance est attribuée à l'arrivée des immigrés économiques, soit, en moyenne, ${ }^{\prime} 5^{\prime} 000$ nouveaux habitants par an entre 1996 et $2001^{17}$. Bien que l'expansion urbaine soit régie par un plan d'aménage-

14 Rolf Steppacher, Pascal van Griethuysen, «Propriété et ressources minérales: la combinaison spécifique de la croissance économique occidentale», Proceedings, Interdisciplinary Workshop on the Institutional Foundations of World Trade, Genève, IUED, 2002, pp. 1-12.

15 Rolf Steppacher, «Ecologie globale...», op. cit.

16 Pascal van Griethuysen, «Rationalité économique et logique de précaution», Revue européenne des sciences sociales, tome XLII, $\mathrm{n}^{\circ}$ 130, 2004, pp. 203-227.

17 Mariana Butrón Oporto, Jorge Miguel Veizaga Rosales, La población en el Municipio Cercado de Cochabamba. Diagnóstico Sociodemográfico por Distritos, Cochabamba, Universidad Mayor de San Simón (UMSS), Centro de Estudios de Población (CEP), Convenio ASDI/UMSS, CEP-UMSS, 2003, p. 9. 
ment du territoire établissant les règles d'urbanisation, la majorité de ces immigrés s'est installée dans des squats situés dans des zones périphériques de Cochabamba ${ }^{18}$.

Le Service municipal d'eau potable et d'assainissement (SEMAPA) n'a pas été en mesure de suivre le rythme de cette croissance urbaine. Ainsi, tant l'exploitation de nouvelles ressources hydriques que l'extension du service d'eau potable vers des zones périphériques sont restées en arrière. Par conséquent, en 2005, seuls $48 \%$ des foyers ont été connectés au service étatique du SEMAPA ${ }^{19}$. Ceux-ci se situent surtout dans les zones résidentielles du nord-est et du centreville et appartiennent essentiellement à des niveaux socio-économiques plus élevés. Bien que raccordés au service étatique, une minorité de ces foyers accède à l'eau potable vingt-quatre heures sur vingt-quatre ${ }^{20}$.

Les raisons pour lesquelles le service étatique n'est pas resté dans la course sont diverses. Le taux de la croissance urbaine a certes posé un problème majeur ${ }^{21}$, notamment dans le contexte de déficit structurel que connaissait le SEMAPA, l'amenant à s'engager dans des relations de crédit. En effet, en 2001, les revenus monétaires du SEMAPA n'ont couvert que $87 \%$ de ses dépenses ${ }^{22}$ et les dettes contractées s'élèvent, en 2005, à 30 millions de dollars ${ }^{23}$. Ce déficit structurel est accompagné d'une gestion inefficiente, de telle sorte que, en 2005, le SEMAPA est accusé de népotisme, d'employer trop de personnel et de disposer d'une administration avant tout bureaucratique ${ }^{24}$. Le taux élevé des pertes physiques et commerciales d'eau potable $(53,4 \% \text { d'eau brute en } 2004)^{25}$ témoigne aussi bien de la situation de déficit structurel que de la gestion inefficiente. $\mathrm{Ce}$ taux élevé est également le signe de l'absence d'une autorité dotée de la volonté et de la capacité d'assurer l'observation des obligations liées à l'accès au service d'eau potable, entraînant entre autres des connexions illégales et des paiements insuffisants.

En conséquence, vu l'impuissance du SEMAPA à rester dans la course, 52\% des foyers, situés surtout dans les zones du nord-ouest et du sud, ont dû trouver des moyens alternatifs d'approvisionnement en eau potable. Appartenant à des niveaux socio-économiques plus défavorisés, la majeure partie de ceux-ci ont recouru à des puits privés ou à des camions-citernes; ces derniers exploitent également l'eau souterraine afin de la distribuer dans les zones soumises au manque d'eau. Une autre partie a constitué des coopératives, des associations ou des comités d'eau, mettant sur pied des services communautaires visant à satisfaire les besoins minimaux des usagers en eau potable.

18 Simon Marvin, Nina Laurie, «An Emerging Logic of Urban Water Management, Cochabamba, Bolivia», Urban Studies, vol. 36, nº 2, 1999, pp. 341-357.

19 Equipo Tecnico Coordinadora del Agua y de la Vida, Temas críticos en la gestion de SEMAPA, Cochabamba, 07/04/2005.

20 Gonzalo Maldonado Rojas, Perspectiva y Futuro de la empresa SEMAPA, La Paz, Fondo Editorial de Diputados, 2001, p. 65.

21 Simon Marvin, Nina Laurie, op. cit.

22 Gonzalo Maldonado Rojas, op. cit., p. 35.

23 Los Tiempos, 19/05/2005.

24 ASICA-SUR, FEDECOR, Coordinadora del Agua y de la Vida, op. cit.

25 Equipo Tecnico Coordinadora del Agua y de la Vida, op. cit. 
Bien qu'il existe une grande diversité parmi ces services communautaires en termes de couverture, d'infrastructure, de continuité, de tarifs, de quantité et de qualité de ressources d'eau (la majorité recourt à des puits communautaires), ainsi qu'en termes de structure interne des organisations, la majorité de celles-ci ont certaines caractéristiques en commun. D'abord, ces services représentent des unités sociales avec des frontières bien définies. Ils reposent sur un mode d'organisation sociale fondé sur les principes de réciprocité et de partage, autrement dit sur un système d'appui mutuel entre usagers, visant la mise en commun de ressources monétaires et non monétaires, ainsi que l'organisation en commun de travaux ${ }^{26}$. De plus, ces services communautaires disposent d'un ensemble de règles collectives qui régissent l'accès, l'usage et la gestion de l'eau, déterminant ainsi les relations entre les usagers concernant cette ressource. A ces règles s'ajoutent d'autres règles collectives qui régulent l'inclusion et l'exclusion des usagers par rapport aux services communautaire ${ }^{27}$. L'observation de l'ensemble de ces règles est assurée par des autorités internes, ce qui entraîne entre autres un faible taux de pertes physiques et commerciales de l'eau potable ${ }^{28}$ et un taux élevé de récupération des coûts investis dans les services fournis ${ }^{29}$.

Bien que les statistiques indiquent approximativement que, en 2001, $10 \%$ de foyers se sont approvisionnés en eau potable à travers les systèmes communautaires $^{30}$, les chiffres réels d'approvisionnement par les différents moyens alternatifs restent mal connus. Les raisons en sont diverses. D'abord, toute tentative d'élaborer des statistiques fiables est déjouée par le fait que les foyers s'approvisionnent souvent à travers plusieurs moyens parallèles, y compris les foyers raccordés au réseau du SEMAPA. Ensuite, cet approvisionnement se déroule avant tout dans l'informalité, rendant la collecte de données précises plus difficile. Cependant, en 2005, les estimations indiquent l'existence de 230 services privés de camions-citernes ${ }^{31}$, ainsi qu'un total de 120 coopératives, associations et comités d'eau dont 40 sont associés à l'Association de systèmes communautaires d'eau de la zone sud de Cochabamba, ASICA-SUR ${ }^{32}$. Contrairement à ces estimations relativement précises, celles concernant le nombre de puits (privés et communautaires) divergent profondément, varient de $3000^{33}$ à $8000^{34}$ pour l'ensemble des zones urbaines.

Les raisons pour lesquelles le nombre de puits n'est pas connu, même approximativement, sont encore plus complexes. Tout d'abord, en 2000, le gouvernement bolivien a voté une loi prohibant le forage de nouveaux puits dans la zone de concession du SEMAPA sans autorisation antérieure (art. 80) ${ }^{35}$. Par ailleurs,

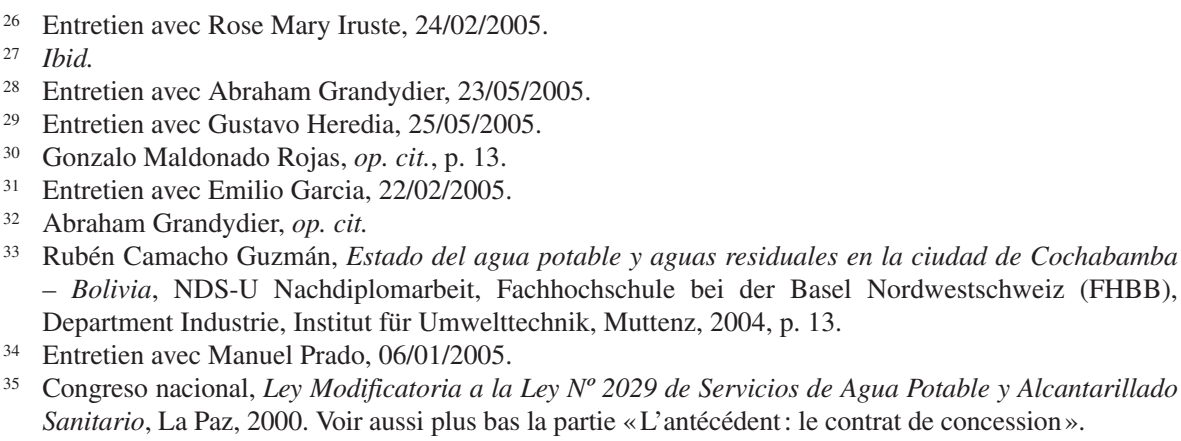


cette loi oblige les propriétaires des terrains bâtis, non bâtis, domestiques ou commerciaux situés dans les zones raccordées au réseau du SEMAPA à s'y connecter, et donc d'abandonner l'approvisionnement en eau potable par des puits privés (art. 76 et 78$)^{36}$. Nonobstant, au cours des cinq dernières années, ces règles sont rarement observées, avec pour effet l'exploitation indiscriminée d'eau souterraine et, en conséquence, la baisse sensible de la nappe phréatique dans l'ensemble du territoire municipal ${ }^{37}$.

Or, il est important de souligner qu'au sein de ce territoire, la nature même de l'aquifère varie d'une zone à l'autre: comme la zone nord constitue la zone de recharge de l'aquifère, les puits qui y sont forgés disposent d'une quantité et d'une qualité d'eau supérieures à celles de ceux du sud, où l'eau est essentiellement classée comme non potable ${ }^{38}$.

Pour conclure, les asymétries entre les zones résidentielles et périphériques de Cochabamba se reflètent donc non seulement dans les moyens d'approvisionnement en eau potable et les niveaux socio-économiques, mais également dans la quantité, la qualité et le prix de l'eau potable. En principe, les usagers des zones périphériques consomment par jour moins d'eau potable que les usagers des zones résidentielles; en outre, ils paient plus cher une eau potable de qualité inférieure: le prix par mètre cube varie entre 0,1 et 0,3 dollar ${ }^{39}$ (service étatique du SEMAPA), entre 0,4 et 0,6 dollar ${ }^{40}$ (services communautaires), et entre 1,9 et 2,5 dollars ${ }^{41}$ (services privés de camions-citernes). Ces asymétries se répercutent directement sur les taux d'espérance de vie et de mortalité enfantine. Dès lors, les zones périphériques présentent les taux d'espérance de vie les plus bas (47 ans contre 67 ans pour une personne née dans une zone résidentielle), ainsi que les taux de mortalité enfantine les plus hauts (145 décès enfantins contre 45 décès enfantins pour 1000 enfants nés dans une zone résidentielle) $)^{42}$.

Il ne fait aucun doute que ces asymétries ont été à l'origine des tensions et des conflits sociaux qui ont donné lieu à de nombreuses propositions visant précisément à atténuer les problèmes d'approvisionnement en eau potable à Cochabamba.

36 Ibid.

37 Entretien avec Ivan Aviles, 19/02/2005; entretien avec Christian Neumann-Redlin, 28/02/2005.

38 Sven Renner, Carlos Velasco, Geología e Hidrología del Valle Central de Cochabamba, Boletín del Servicio Nacional de Geología y Minería, no 34, Cochabamba, 2000, 105 p.

39 Servicio Municipal de Agua Potable y Alcantarillado (SEMAPA), Contrato de Concesión SEMAPASISAB, Cochabamba, 2002.

40 David Fernández Rojas, Evaluación de la gestión de recursos hídrico en cooperativas y asociaciones de suministro de agua potable en la zona de Valle Hermoso-Cochabamba, tesis de maestría, Cochabamba, CLAS, ITC, UMSS, IHEE-Delft, 2000.

41 Entretien avec Emilio Garcia, 22/02/2005.

42 Carmen Ledo Garía, Urbanisation and Poverty in the Cities of the National Economic Corridor in Bolivia: Case Study: Cochabamba, Delft, Delft University Press, 2002, 263 p. 
L'une de ces propositions était de mettre en place un partenariat public-privé, en déléguant l'exploitation et la distribution de l'eau potable à une organisation privée. En effet, en septembre 1999, le gouvernement bolivien a signé un contrat de concession de quarante ans avec le consortium multinational «Aguas del Tunari » et a voté, peu après, une nouvelle loi visant à réglementer le secteur des services d'eau potable et d'assainissement.

Etant donné que ce partenariat a déjà fait l'objet de nombreuses études ${ }^{43}$, l'article n'entrera pas ici dans les détails. Néanmoins, il est important de souligner que tant la signature du contrat que le vote de la nouvelle loi ont déclenché des mobilisations sociales sans précédent qui ont culminé, en 2000, dans ce qui est connu comme la «guerre de l'eau». Face aux conflits, le gouvernement a dû céder aux demandes sociales: il a résilié le contrat de concession et a retransféré l'exploitation et la distribution de l'eau potable au SEMAPA. De plus, il a abrogé la nouvelle loi, en la remplaçant par une autre qui régule actuellement le secteur des services d'eau potable et d'assainissement en Bolivie. Cette loi reconnaît explicitement les droits des organisations communautaires de s'approvisionner en eau potable au sein d'une concession donnée par l'Etat à une autre organisation, qu'elle soit étatique ou privée.

\section{La perspective: la cogestion étatique-communautaire ${ }^{44}$}

En 2004, quatre ans après la «guerre de l'eau», diverses organisations (le SEMAPA, l'entreprise privée Plastiforte, l'ONG ProHabitat, l'ASICA-SUR et d'autres organisations communautaires de la zone sud) ont constitué un nouveau partenariat afin de faire face à la situation toujours défavorisée de la zone sud en termes de services d'eau potable. Ce partenariat vise à relier le service étatique à des services communautaires en impulsant dans cette zone une «nouvelle» modalité de gestion commune: la cogestion étatique-communautaire.

Avant d'entrer dans les détails, il est opportun de mentionner que Marvin et Laurie $^{45}$ indiquent que, entre 1988 et 1996, la Banque mondiale, à travers le Fonds social d'urgence (plus tard Fonds social d'investissement, FIS), a financé un programme orienté vers le développement de stratégies visant l'extension du réseau d'eau potable du SEMAPA à des zones jusque-là non connectées. Selon ces auteurs, les usagers y ont joué un rôle décisif concernant l'installation et la gestion locale de réseaux secondaires. Afin d'intégrer «les communautés dans

43 Willem Assies, «David versus Goliath in Cochabamba: Water Rights, Neoliberalism, and the Revival of Social Protest in Bolivia», Latin American Perspectives, Issue 130, vol. 30, n 3, 2003, pp. 14-36; Carmen Peredo, Carlos Crespo, Omar Fernández, Los Regantes de Cochabamba en la Guerra del Agua. Presión social y negociación, Cochabamba, CESU-UMSS, Memoria Editores, 2004, 220 p.; Andrew Nickson, Claudia Vargas, «The Limitations of Water Regulation: The Failure of the Cochabamba Concesión in Bolivia», Bulletin of Latin American Research, vol. 21, n 1, 2002, pp. 99-120.

44 Cette partie a été nécessairement soumise à des simplifications. Les relations entre les différents partenaires qui cherchent à approvisionner les usagers en eau potable sont naturellement beaucoup plus complexes que ne le montre l'évocation qui suit.

45 Simon Marvin, Nina Laurie, op. cit., pp. 351-352. 
tous les aspects de la gestion locale $»^{46}$, ce programme a en outre mis sur pied différentes coopératives.

Malheureusement, les auteurs n'explicitent ni le nombre de coopératives effectivement créées dans le cadre de ce programme, ni la modalité de gestion locale préconisée par le SEMAPA et les communautés. Ils indiquent cependant que ce programme s'est terminé en 1996 lorsque le financement canalisé par le FIS a été suspendu.

Bien qu'il reste encore des questions ouvertes au sujet de ce programme, on peut désormais douter de la «nouveauté » de la modalité de cogestion envisagée actuellement à Cochabamba. Jusqu'à présent, cette modalité comprend pour le SEMAPA : 1) la prolongation des lignes principales du système centralisé jusqu'aux points d'entrée des réseaux secondaires de chacune des organisations communautaires ; 2) la vente de l'eau potable «en bloc» aux différentes organisations (cela signifie que le SEMAPA fournit une quantité d'eau fixe, tandis que les organisations communautaires ont la charge de la distribuer). Pour ces dernières, cette modalité englobe: 1) l'installation de réseaux secondaires dans le cas où ces réseaux n'existeraient pas encore (cela se réalise dans le cadre du programme AGUA TUYA avec le soutien de l'entreprise privée Plastiforte, qui fournit les matériaux nécessaires et offre l'assistance technique ${ }^{47}$, ainsi qu'à l'aide de l'ONG ProHabitat, qui accorde des microcrédits aux communautés ${ }^{48}$ ); 2) la gestion de l'eau au sein de chaque organisation communautaire.

Les droits et les obligations particuliers liés à cette modalité de cogestion font, depuis 2004, l'objet de négociations entre le SEMAPA et les différentes organisations communautaires ${ }^{49}$. Bien que ces négociations ne soient pas encore achevées, la modalité préconisée à ce jour peut constituer une alternative à celles communément envisagées : d'une part, elle met en relief les potentiels aussi bien des régimes de propriété que des régimes de possession, tout en permettant de faire face aux contraintes particulières liées aux premiers (solvabilité, rentabilité); de l'autre, elle relie les différentes logiques auxquelles le SEMAPA et les organisations communautaires sont soumis. En effet, puisque le SEMAPA finance ses activités économiques avant tout par des crédits, il est nécessairement guidé par la rationalité économique spécifique des régimes de propriété. Contrairement au SEMAPA, les organisations communautaires, en fondant l'approvisionnement en eau potable sur les principes de réciprocité et de partage, sont surtout régies par la raison écosociale propre aux régimes de possession.

Or, la combinaison entre ces différentes logiques a des avantages aussi bien pour le SEMAPA que pour les organisations communautaires: le fait que le SEMAPA prolonge, opère et maintient seulement les lignes principales et non les réseaux secondaires diminue considérablement les coûts d'extension des services d'eau potable vers les zones périphériques du sud. En opérant et maintenant les seules lignes principales, les pertes physiques et commerciales d'eau potable deviennent désormais plus contrôlables, entraînant, en principe, une réduction des pertes d'eau potable non facturée, et ainsi une maximisation des

\footnotetext{
46 Ibid.

47 Entretien avec Gustavo Heredia, 25/05/2005.

48 Entretien avec Antonia Terrazas et Mona Achi, 23/02/2005.

49 Entretien avec Juan José Salinas, 24/02/2005.
} 
revenus monétaires pour le SEMAPA. Cette maximisation est favorisée par le fait que les pertes produites au sein des réseaux secondaires restent sous le contrôle des organisations communautaires, qui, d'ailleurs, se montrent plus efficaces pour faire face à celles-ci. Finalement, le fait que le SEMAPA vend l'eau potable «en bloc» aux organisations communautaires, en concluant pour ce faire un seul contrat avec chaque organisation et non avec chaque usager, réduit notablement les coûts d'administration. En conséquence, la modalité de cogestion ainsi préconisée rend possibles pour le SEMAPA à la fois la réduction des coûts et la maximisation des revenus monétaires, tout en lui permettant de faire face aux contraintes de la solvabilité et de la rentabilité (en vue de rembourser les crédits contractés).

En principe, tant la réduction des coûts que la maximisation des revenus se reflètent dans le prix d'eau potable vendue «en bloc» aux organisations communautaires. Ainsi, en plus de recevoir une eau potable d'une qualité supérieure à celle de l'eau exploitée jusque-là, les organisations communautaires ont l'avantage de l'acquérir à un prix inférieur à celui de l'eau perçu actuellement. En outre, le fait de recevoir l'eau potable du SEMAPA «en bloc» leur permet d'assurer une demande globalement solvable tout en bénéficiant de la souplesse d'une organisation sociale fondée sur la réciprocité et le partage. Ainsi, conformément aux systèmes d'appui mutuel, cette modalité permet aux usagers les plus défavorisés de s'acquitter de leur consommation d'eau potable sous forme de travaux, autrement dit en termes non monétaires. Ces travaux peuvent être liés aussi bien à l'installation, à l'opération ou à la maintenance des réseaux secondaires d'eau potable qu'à d'autres activités relatives à la vie en commun. Les contributions réalisées sous forme de travaux doivent nécessairement être compensées par celles en termes monétaires versées par d'autres usagers de services communautaires, de telle sorte que les organisations elles-mêmes demeurent solvables envers le SEMAPA. En définitive, le fait que les usagers se réunissent et concluent un seul contrat avec le SEMAPA permet aux organisations communautaires de manifester envers ce service une demande solvable, tandis qu'au sein des organisations, les contributions peuvent s'effectuer par des moyens monétaires et non monétaires. Les règles internes qui guideront ces dernières demanderont à être précisées à la lumière du contexte spécifique de chaque service communautaire et feront l'objet de recherches futures.

Pour conclure, la modalité de cogestion étatique-communautaire de l'eau potable peut constituer une alternative aux modalités de gestion communément envisagées. Nonobstant, le succès de cette modalité dépend surtout de la volonté et de la capacité des autorités concernées à la mettre en œuvre, et à assurer subséquemment l'observation des droits et des obligations qui y sont liés. Il dépend également des capacités du SEMAPA à faire face aux contraintes de la solvabilité et de la rentabilité afin de rembourser les crédits contractés, ainsi que des capacités des communautés à gérer les services communautaires selon les principes de réciprocité et de partage, tout en demeurant globalement solvables envers le fournisseur étatique de l'eau potable. 


\section{Bibliographie}

ASICA-SUR, FEDECOR, CoORdinadora del Agua y de la Vida, Conclusiones del Taller de Diagnóstico de SEMAPA, Cochabamba, Carta al Directorio de SEMAPA, 13/05/2005.

Assies, Willem, «David versus Goliath in Cochabamba: Water Rights, Neoliberalism, and the Revival of Social Protest in Bolivia», Latin American Perspectives, Issue 130, vol. 30, $\mathrm{n}^{\circ}$ 3, 2003 , pp. 14-36.

Bromley, Daniel, Economic Interests and Institutions: The Conceptual Foundations of Public Policy, Oxford, Blackwell, 1989.

—, Environment and Economy: Property Rights and Public Policy, Oxford, Blackwell, 1991.

Butrón Oporto, Mariana, Veizaga Rosales, Jorge Miguel, La población en el Municipio Cercado de Cochabamba. Diagnóstico Sociodemográfico por Distritos, Cochabamba, Universidad Mayor de San Simón (UMSS), Centro de Estudios de Población (CEP), Convenio ASDI/UMSS, CEPUMSS, 2003, 68 p.

CAMACHO GuZMÁN, Rubén, Estado del agua potable y aguas residuales en la ciudad de Cochabamba - Bolivia, NDS-U Nachdiplomarbeit, Fachhochschule bei der Basel Nordwestschweiz (FHBB), Department Industrie, Institut für Umwelttechnik, Muttenz, 2004, 58 p.

Congreso nacional, Ley Modificatoria a la Ley $N^{o} 2029$ de Servicios de Agua Potable y Alcantarillado Sanitario, La Paz, 2000.

Equipo Tecnico Coordinadora del Agua y de la Vida, Temas críticos en la gestion de SEMAPA, Cochabamba, 07/04/2005.

FERNÁNDEZ ROJAS, David, Evaluación de la gestión de recursos hídrico en cooperativas y asociaciones de suministro de agua potable en la zona de Valle Hermoso-Cochabamba, tesis de maestría, Cochabamba, CLAS, ITC, UMSS, IHEE-Delft, 2000.

GRIETHUYSEN, Pascal (VAN), «Rationalité économique et logique de précaution», Revue européenne des sciences sociales, tome XLII, $\mathrm{n}^{\circ}$ 130, 2004, pp. 203-227.

Heinsohn, Gunnar, SteIGer, Otto, Eigentum, Zins und Geld. Ungelöste Rätsel der Wirtschaftswissenschaften, Marburg, Metropolis, 2002 [1996].

Ledo GaRíA, Carmen, Urbanisation and Poverty in the Cities of the National Economic Corridor in Bolivia: Case Study: Cochabamba, Delft, Delft University Press, 2002, 263 p.

Maldonado Rojas, Gonzalo, Perspectiva y Futuro de la empresa SEMAPA, La Paz, Fondo Editorial de Diputados, 2001, 98 p.

Marvin Simon, Laurie, Nina, «An Emerging Logic of Urban Water Management, Cochabamba, Bolivia», Urban Studies, vol. 36, nº 2, 1999, pp. 341-357.

Nickson, Andrew, VArgas, Claudia, "The Limitations of Water Regulation: The Failure of the Cochabamba Concesión in Bolivia», Bulletin of Latin American Research, vol. 21, $\mathrm{n}^{\circ}$ 1, 2002, pp. 99-120.

Peredo, Carmen, CRespo, Carlos, Fernández, Omar, Los Regantes de Cochabamba en la Guerra del Agua. Presión social y negociación, Cochabamba, CESU-UMSS, Memoria Editores, 2004, 220 p.

Renner, Sven, Velasco, Carlos, Geología e Hidrología del Valle Central de Cochabamba, Boletín del Servicio Nacional de Geología y Minería, no 34, Cochabamba, 2000, 105 p.

SCHLAGER, Edella, Ostrom, Elinor, «Property-Rights Regimes and Natural Resources: A Conceptual Analysis », Land Economics, vol. 68, n 3, 1992, pp. 249-262.

Servicio Municipal de Agua Potable y Alcantarillado (SEMAPA), Contrato de Concesión SEMAPA-SISAB, Cochabamba, 2002.

STEPPACHER, Rolf, «Ecologie globale et développement», notes de cours, 14/12/2000, 18 et 25/01/2001, Genève, IUED, semestre d'hiver 2003/2004.

—, «La petite différence et ses grandes conséquences: possession et propriété», entretien avec Rolf Steppacher, in Comeliau, Christian (dir.), Brouillons pour l'avenir. Contribution au débat sur les alternatives, Nouveaux Cahiers de l'IUED, nº 14, Genève, IUED; Paris, Presses Universitaires de France, 2003, pp. 181-190.

—, «La résistible imprécision des notions économiques», in Comeliau, Christian (dir.), L'économie à la recherche du développement. Crise d'une théorie, violence d'une pratique, Nouveaux Cahiers de 1'IUED, n 5, Genève, IUED; Paris, Presses Universitaires de France, 1996, pp. 39-52.

STEPPACHER, Rolf, GRIETHUYSEN, Pascal (VAN), «Propriété et ressources minérales: la combinaison spécifique de la croissance économique occidentale », Proceedings, Interdisciplinary Workshop on the Institutional Foundations of World Trade, Genève, IUED, 2002, pp. 1-12.

TAPIA, Luis, HofFMANN, Sabine, Viaña, Jorge, Rozo, Bernardo, La (re)construcción de lo público : Movimiento social, ciudadanía y gestión de agua en Cochabamba, La Paz, Muela del Diablo, 2005 (à paraître). 


\section{Presse}

Los Tiempos, 18/05/2005, 19/05/2005.

\section{Entretiens}

AviLES, Ivan, coordinateur de forage des puits du Service national de géologie et de minières (SERGEOMIN), Cochabamba, 19/02/2005.

GARCIA, Emilio, conducteur de camion-citerne et ex-secrétaire du syndicat Manantial Tunari, auquel 30 des 230 camions-citernes sont affiliés, Cochabamba, 22/02/2005.

GRANDYDIER, Abraham, président de l'Association des systèmes communautaires d'eau de la zone sud (ASICA-SUR), Cochabamba, 23/05/2005.

HEREDIA, Gustavo, directeur du programme AGUA TUYA, Cochabamba, 25/05/2005, <www.aguatuya. com>.

IRUSTE, Rose Mary, coordinatrice du projet «Habitat para la Mujer Maria Auxiliadora», 24/02/2005, $<$ www.nccr-north-south.unibe.ch>.

Neumann-Reduin, Christian, ex-directeur du projet CABAS (Accord aléman-bolivien de l'eau souterraine), Cochabamba, 28/02/2005.

PRADO, Manuel, ex-directeur du Département de planification du Gouvernement municipal de Cochabamba, Cochabamba, 06/01/2005.

SALINAS, Juan José, coordinateur de l'extension du service d'eau potable du SEMAPA dans la zone sud, 24/02/2005.

TERRAZAS, Antonia, ACHI, Mona, conseillères de l'ONG ProHabitat, 23/02/2005.

\section{Groupe de travail}

STEPPACHER, Rolf, groupe de travail «Propriété et possession», séance du 05/05/2005, Genève. 